\title{
TV/Series
}

14 | 2018

Posthumains en séries

\section{« Have you ever questioned the nature of your reality?»: Westworld (HBO, 2016-) et la culture du complot}

Sébastien Lefait

\section{OpenEdition}

Journals

Édition électronique

URL : http://journals.openedition.org/tvseries/2949

DOI : 10.4000/tvseries.2949

ISSN : 2266-0909

Éditeur

GRIC - Groupe de recherche Identités et Cultures

Référence électronique

Sébastien Lefait, « « Have you ever questioned the nature of your reality? » : Westworld (HBO, 2016-) et la culture du complot », TV/Series [En ligne], 14 | 2018, mis en ligne le 31 décembre 2018, consulté le

24 avril 2019. URL : http://journals.openedition.org/tvseries/2949; DOI : 10.4000/tvseries.2949

Ce document a été généré automatiquement le 24 avril 2019.

\section{(c) (i) () $९$}

TV/Series est mis à disposition selon les termes de la licence Creative Commons Attribution - Pas d'Utilisation Commerciale - Pas de Modification 4.0 International. 


\title{
« Have you ever questioned the nature of your reality? »: Westworld (HBO, 2016-) et la culture du complot
}

\author{
Sébastien Lefait
}

1 Comme un grand nombre de fictions actuelles, Westworld s'appuie sur la popularité des théories du complot ${ }^{1}$. Dans Conspiracy Theories: Secrecy and Power in American Culture, Mark Fenster définit la théorie du complot comme « la conviction qu'un individu ou un groupe d'individus omnipotent(s) contrôle(nt) en secret tout ou partie de l'ordre politique et social ${ }^{2}$. " Le terme clé de cette définition est bien sûr le « secret ", qui implique que tout récit complotiste repose sur une logique du visible et de l'invisible, et comprenne un certain nombre de dévoilements ou de révélations.

2 Cependant, alors que les recettes du récit complotiste, qui tendent vers le fictionnel mais trouvent leur origine dans une lecture paranoïaque d'événements historiques réels, ont été abondamment décrites, il n'en va pas de même pour leur réception. Plus particulièrement, l'impact de la culture du complot sur ceux et celles qui la consomment reste très peu étudié. C'est donc dans le but de combler ces manques que je m'intéresserai ici aux effets de style conspirationnistes de Westworld, mais également à leurs conséquences. En effet, la série ne se contente pas de s'inscrire dans la culture du complot, mais en tire parti pour fidéliser ses spectateurs, et encourage chez ces derniers des réflexes paranoïaques dont on se demandera dans quelle mesure ils peuvent dépasser les limites spatiales et temporelles de la fiction sérielle pour persister dans le monde réel.

\section{Westworld, un récit complotiste}

3 A priori, identifier en Westworld un récit complotiste n'a rien de très original. D'abord parce que la série n'est pas la seule à recycler les stratégies habituelles de ce que de nombreux auteurs considèrent comme un genre fictionnel à part entière. On ne compte 
plus les productions télévisées qui, à l'image de 24 (Fox, 2001-2010 ; 24 heures chrono, en France) ou Homeland (Showtime, 2011-), font reposer leur technique narrative sur la possibilité qu'un attentat terroriste soit en préparation, ou, à l'image de Lost ( $A B C$, 2004-2010), tirent leur popularité d'une exigence envers leur public: celle de percer le mystère d'un univers opaque mais, fort heureusement, truffé d'indices ${ }^{3}$. Ensuite parce que, comme l'assassinat de Kennedy et le 11 septembre 2001 (qui sont certes des événements réels), Westworld est une « série-événement " qui a immédiatement suscité des lectures complotistes à la fois très explicites et très largement médiatisées. Dès la diffusion du premier épisode, la réception de la série s'est inscrite dans le cadre d'un rapprochement entre les stratégies narratives de ses concepteurs et celles du genre complotiste ${ }^{4}$.

Rien d'étonnant, là encore, à ce que les premières critiques de la série soient unanimes pour y trouver les traces de la culture du complot. De fait, les stratégies éprouvées du récit conspirationniste sont facilement repérables dans la première saison de Westworld. Je commencerai par deux traits récurrents dans ce type de récit, non pas parce que ce sont les plus évidents, mais parce que je les évoque dans un autre article figurant dans le présent numéro et intitulé " "These violent delights have violent ends", Apocalyptic remediation, or, can there be a Revelation in this (West)world? ». Le premier de ces deux traits caractéristiques est la parenté entre les récits complotistes et les récits apocalyptiques, qui convoquent un imaginaire de la fin dont on peut considérer qu'il est l'un des enjeux principaux de Westworld'. C'est ce que met en évidence la citation de Shakespeare choisie dans le titre de cet article, qui fait de l'intrigue de la série une progression mécanique vers une fin violente, désastre de proportions bibliques à la mesure des plaisirs proposés dans le parc. Le second est une importante dimension référentielle et auto réflexive, que j'ai décrite en analysant l'utilisation de citations shakespeariennes dans les dialogues de la série, pour la référentialité ${ }^{6}$, et en identifiant un certain nombre de répliques métaleptiques, pour ce qui concerne l'autoréflexivitét.

En plus de ces deux éléments génériques, la littérature universitaire sur le récit complotiste associe généralement sa popularité à son caractère plaisant et addictif, plein de suspense, de rebondissements, de révélations, de masques qui tombent, de découvertes qui donnent accès à une vérité a priori cachée, ces derniers éléments s'enchaînant de préférence rapidement ${ }^{8}$. Il est évident que Westworld inclut tous ces ingrédients, qu'elle a en commun avec beaucoup d'autres séries, mais dont elle fait un argument plus efficace pour attirer les spectateurs. C'est du moins ce que l'on peut penser si l'on en juge par ses taux d'audience record, sans précédent pour HBO et dépassant même, selon certaines estimations, ceux de Game of Thrones 9 .

Westworld tire surtout cet avantage du comportement complotiste de ses personnages. Le plus souvent, le protagoniste de ce type de récit dévoile un secret ${ }^{10}$, qui le mène à une série d'opérations cognitives logiques, fondées sur un lien causal. Les déductions interviennent en cascade pour former une séquence temporelle et déboucher sur un dénouement heureux ${ }^{11}$. Ce dénouement consiste le plus souvent à se libérer du joug d'un pouvoir centralisé, représenté par un grand maître invisible, ou par des sociétés secrètes ${ }^{12}$. Dans la première saison, cette suite d'actions correspond quasiment exactement au cheminement de Maeve, et à travers elle de toute la communauté des "hôtes ». Pour Maeve, tout part d'un souvenir qu'elle n'aurait pas dû avoir, celui d'une de ses nombreuses morts, en compagnie de sa fille. Désireuse de comprendre d'où lui vient ce souvenir et d'où elle vient en tant qu'individu, elle demande à un autre robot, Hector, de 
lui ouvrir le ventre, ce qui lui permet de découvrir un fragment de balle à l'intérieur de son corps, signe qu'elle est revenue d'entre les morts. Tirant les conséquences de cette preuve, elle imagine un moyen d'interagir avec les techniciens de maintenance du parc, Felix et Sylvester, puis d'obtenir qu'ils augmentent sa capacité de prise de décision, ce qui lui permet de découvrir l'existence de l'entreprise Delos et de ses employés. Elle entreprend alors de les combattre, jusqu'à la libération qui semble pouvoir être la sienne à la fin de la première saison.

7 On peut également considérer que le mystérieux homme en noir passe par des étapes similaires. À la lecture de certains indices, et notamment après avoir trouvé, à de nombreux endroits différents du parc, le même symbole représentant un labyrinthe, il décide de trouver le niveau caché de Westworld, et d'en dépasser les limites, comme s'il s'agissait d'un jeu vidéo grandeur nature. Sa quête, contrairement à celle de Maeve, tire son intérêt de ne jamais aboutir, car son achèvement mettrait fin à sa recherche du plaisir que semble lui procurer l'investigation. On peut en prendre pour preuve sa déception lorsqu'il pense avoir enfin trouvé le centre du labyrinthe, qui fait depuis si longtemps l'objet de son obsession : «Alors c'est ça, le centre du labyrinthe ${ }^{13}$ ? ", déclaret-il. Mais à sa grande satisfaction, Dolores, alors présente à ses côtés, lui répond par une réplique énigmatique qui relance le mystère et suscite à nouveau son intérêt : « La fin se déroule dans un endroit où je ne suis jamais allée, où je fais quelque chose que je ne ferai jamais ${ }^{14}$. » Une grande partie des répliques de l'homme en noir évoque d'ailleurs un jeu de piste interminable, comme quand il demande à Ford : «Comment est-ce que je m'en sors, Robert? Je me rapproche de ce que je cherche ?». Une nouvelle fois, la réponse a pour but d'entretenir le suspense, pour l'homme en noir comme pour les spectateurs : «Je ne sais pas. Qu'est-ce que vous cherchez ${ }^{15}$ ?"

Maeve et l'homme en noir s'écartent des personnages-type du western sur plusieurs points, dont le principal est la tâche transdiégétique qui leur est assignée : transférer leur perception du parc aux spectateurs pour décupler le potentiel de la série en lui appliquant un prisme complotiste. Ils se doublent d'enquêteurs que les téléspectateurs sont appelés à imiter lors d'un visionnage attentif de la série. À travers eux, Westworld interpelle constamment son public, en l'invitant notamment à prêter attention au moindre détail. Cela passe également par le personnage de Dolores, à qui l'on pose sans cesse la même question, «Est-ce que tu t'es déjà dit que ton existence était un mensonge ? ${ }^{16}{ }$. Une telle insistance laisse penser qu'on lui cache quelque chose, ce qui constitue en soi une manière d'inciter le spectateur à décrypter, en lieu et place du personnage, l'univers narratif de Westworld. En effet, pour les tenants des théories du complot, toute stratégie visant à inhiber le désir de percer les apparences paraît immédiatement suspecte - la question posée et reposée à Dolores sert à vérifier qu'elle ne se doute de rien quant à la véritable nature du parc ${ }^{17}$. L'interprétation est donc potentiellement sans fin, puisque c'est tout une réalité parallèle qu'il faut décoder, en refusant une lecture conventionnelle pour lui substituer une recherche du sens caché, un dépassement du trompe-l'œil qu'illustre ici le terme « question ». C'est ce qu'exprime également, beaucoup plus directement que Dolores, l'une des hôtesses qui fait entrer William dans le parc lors de sa première visite, quand elle lui déclare que « la moitié du plaisir [du parc] consiste à essayer d'en comprendre le fonctionnement ${ }^{18} »$. 


\section{Westworld, incitation à la réception complotiste}

On peut donc estimer qu'en tant que récit complotiste, Westworld exige des spectateurs une réception sceptique, calquée sur celle de l'homme en noir, de Maeve, de Dolores, et d'autres personnages encore. La spécificité de la série tient ainsi à la manière dont elle cherche à contrôler, de manière parfois assez peu subtile, sa propre réception en provoquant, chez un grand nombre de spectateurs, cette lecture paranoïaque qui s'apparente par conséquent à un réflexe conditionné. Ce réflexe s'avère d'autant plus facile à déclencher qu'il fait appel à une intelligence spectatorielle qui n'est pas, contrairement à celle des robots du parc, artificielle. La série s'inscrit donc parfaitement dans la tendance décrite par Jagodzinski, pour qui la montée en puissance du posthumain se traduit, dans la culture populaire, par une paranoïa généralisée se manifestant dans des scénarios où la machine prend le pouvoir ${ }^{19}$.

Cependant, même si on la remarque de suite, il est difficile de mesurer cette capacité de la série à induire une réception conspirationniste. Je propose de m'y essayer, sans pour autant me livrer à un décryptage de l'œuvre : les aficionados de Westworld le font très bien dans les vidéos explicatives que l'on trouve sur internet. Prenant acte de la concurrence qu'opposent ces amateurs aux professionnels de l'analyse sérielle, j'étudierai donc l'une de ces vidéos, choisie parmi les plus visionnées sur YouTube, pour y détecter les symptômes d'une lecture complotiste. Diffusée le 8 décembre 2016, soit moins d'une semaine après la diffusion du dixième et dernier épisode de la première saison, cette vidéo est sobrement intitulée « Westworld Ending Explained ${ }^{20}$ ».

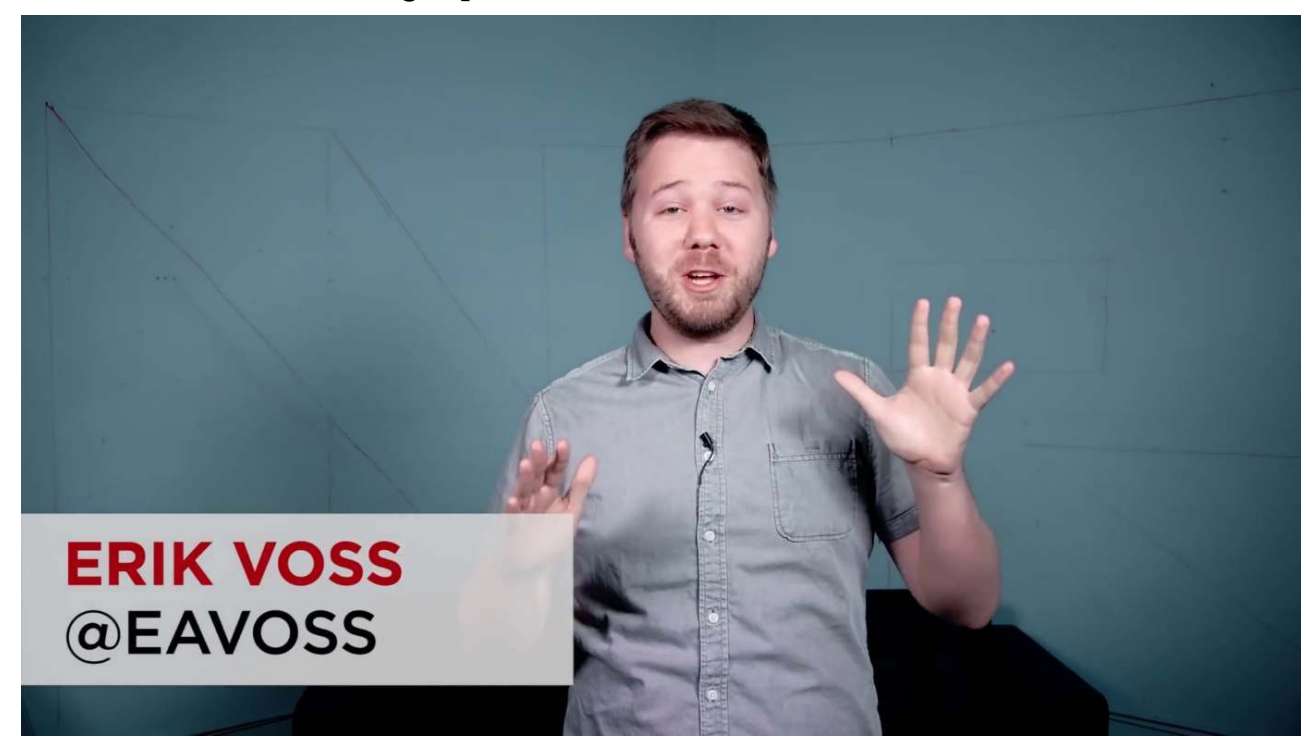

Fig. 1 : Erik Voss : La fin de Westworld pour les nuls...

Le vidéaste amateur, Erik Voss, s'adresse de manière jouissive à une communauté virtuelle, celle des fans de Westworld (Fig. 1). Il illustre, par sa pratique - qui consiste à partager une interprétation personnelle à l'échelle mondiale - un aspect essentiel de la réception complotiste, à savoir une dimension participative qui lui donne un côté jouissif. Voss apparait de suite comme l'archétype des jeunes gens de la génération postindustrielle que décrit Jagodzinski. Selon cet auteur, ils sont caractérisés par une propension à mettre à profit leur connaissance des technologies numériques et leur 
habileté médiatique pour compenser l'angoisse de vivre dans un monde soumis à l'incertitude, en trouvant et partageant entre eux des réponses à certaines questions existentielles qu'ils s'autorisent, par un biais cathartique, à faire porter sur un univers fictionnel ${ }^{21}$. Voss diffuse un remontage explicatif de certains moments de la première saison ${ }^{22}$, qui consiste à faire rentrer des événements complexes dans un schéma qui, de la sorte, apparaît quasiment simpliste. C'est ce que souligne Voss quand il dit qu'il ne s'attendait pas à ce que J. J. Abrams parsème sa série d'un si grand nombre d'indices (Fig. $2)^{23}$.

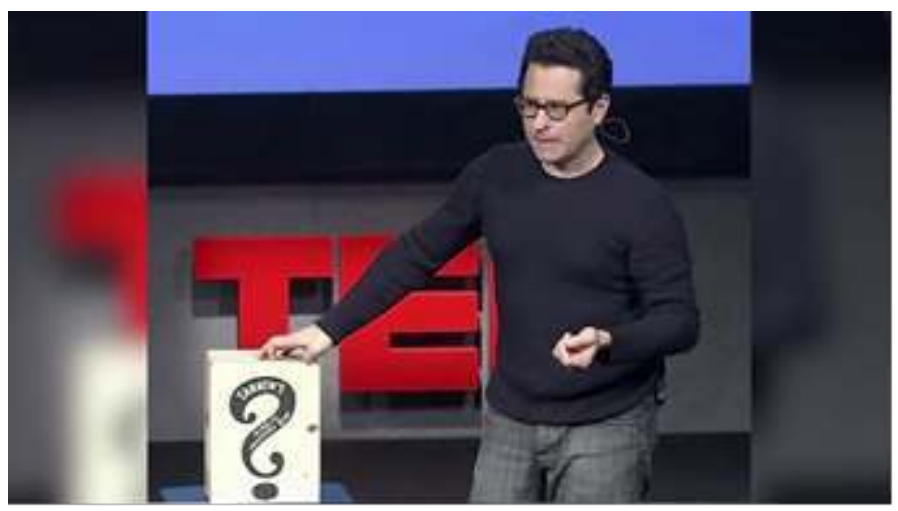

Fig. 2 : J. J. Abrams et sa boîte à secrets

Ce faisant, Voss analyse autant ce qui se trouve dans la série que ce qu'il s'attendait à y trouver. Il incarne une version alternative du «lecteur/spectateur idéal ${ }^{24}$ » dont, dans une théorie du récit plus classique, l'auteur imagine la réaction pour concevoir son œuvre. Mais malgré cette abondance de clés offertes aux spectateurs, Voss cultive une réception obsessionnelle. Il montre même une certaine tendance à l'incohérence et à l'excès, en conformité avec ces caractéristiques pathologiques souvent attribuées à ceux en qui il voit le public privilégié de Westworld: les défenseurs de théories du complot en tous genres ${ }^{25}$. Il s'adresse d'ailleurs directement à eux quand sa liste de révélations atteint son apogée : "Vous avez entendu ça ? C'était le bruit de milliers de défenseurs des théories du complot qui s'excitent tous en même temps ${ }^{26}$ ".

Néanmoins, il ne lui suffit pas d'avoir obtenu clairement des réponses à toutes ses questions. Elles sont arrivées à un rythme trop rapide, ce qui nécessite de repasser en revue les moments importants de la saison écoulée, pour y localiser des détails à première vue insignifiants mais qui, correctement interprétés, auraient permis aux spectateurs d'accéder bien plus tôt au sens global de l'univers narratif de Westworld. Au fil de la vidéo, Voss propose donc un remontage d'indices (en forme de parcours fléché) du sens de la série, lequel n'est accessible qu'au spectateur avisé qu'il prétend incarner (Fig. 32) 


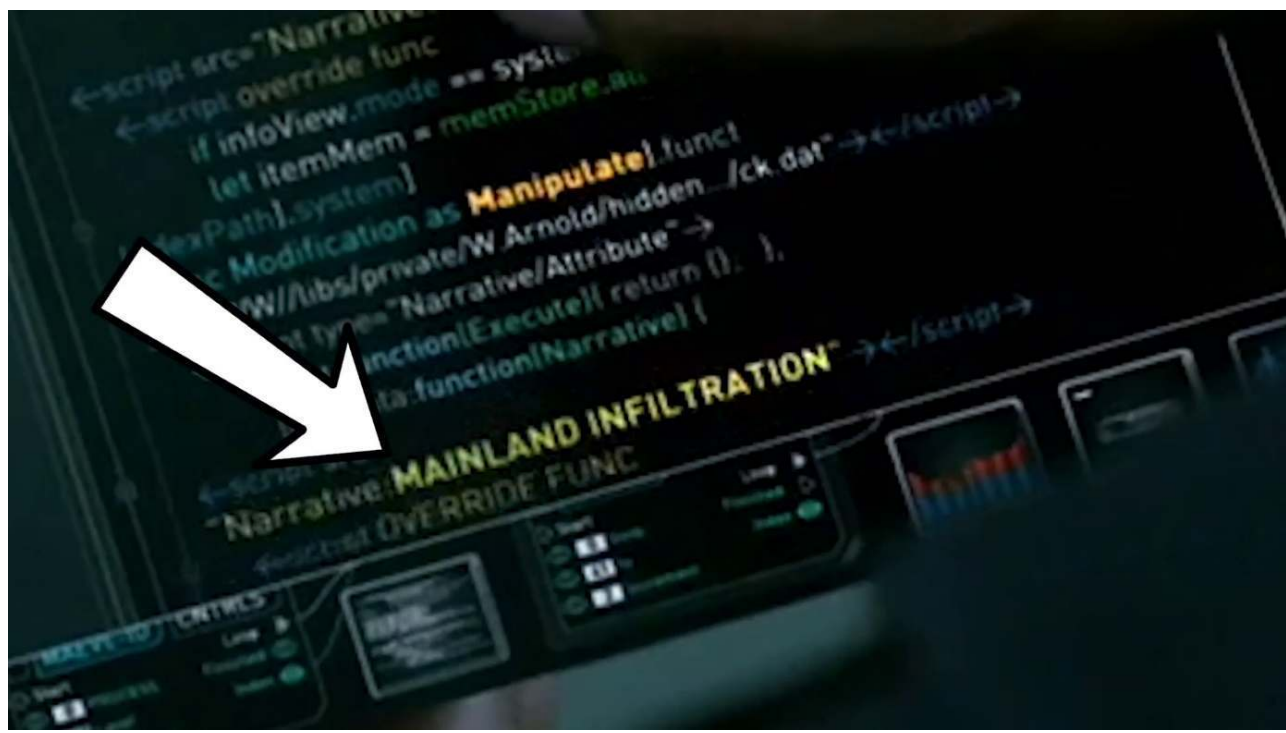

Fig. 3 : Parcours fléché 1 : le sens de l'indice

14 Par cette reconstruction a posteriori, Voss se compte de facto parmi une élite dont les attentes définissent en quoi la série aurait été plus réussie si elle avait plus savamment distillé ses indices. Cela sous-entend que les concepteurs de Westworld ont sous-estimé la capacité de décodage de leur « spectateur idéal ».

Voss propose une telle interprétation à propos du plan où l'on voit Ford diriger des travaux d'excavation pour retrouver la ville enfouie d'Escalante ${ }^{28}$, arguant qu'il permet de deviner quel est son projet (Fig. 4). Cette analyse propose également une image de l'attitude attendue du spectateur: celle d'un légiste ou d'un archéologue susceptible de creuser la surface du visible afin d'y chercher les vestiges d'une réalité cachée, et d'y faire des découvertes qui induisent une relecture de ce qui précède selon de nouvelles normes.

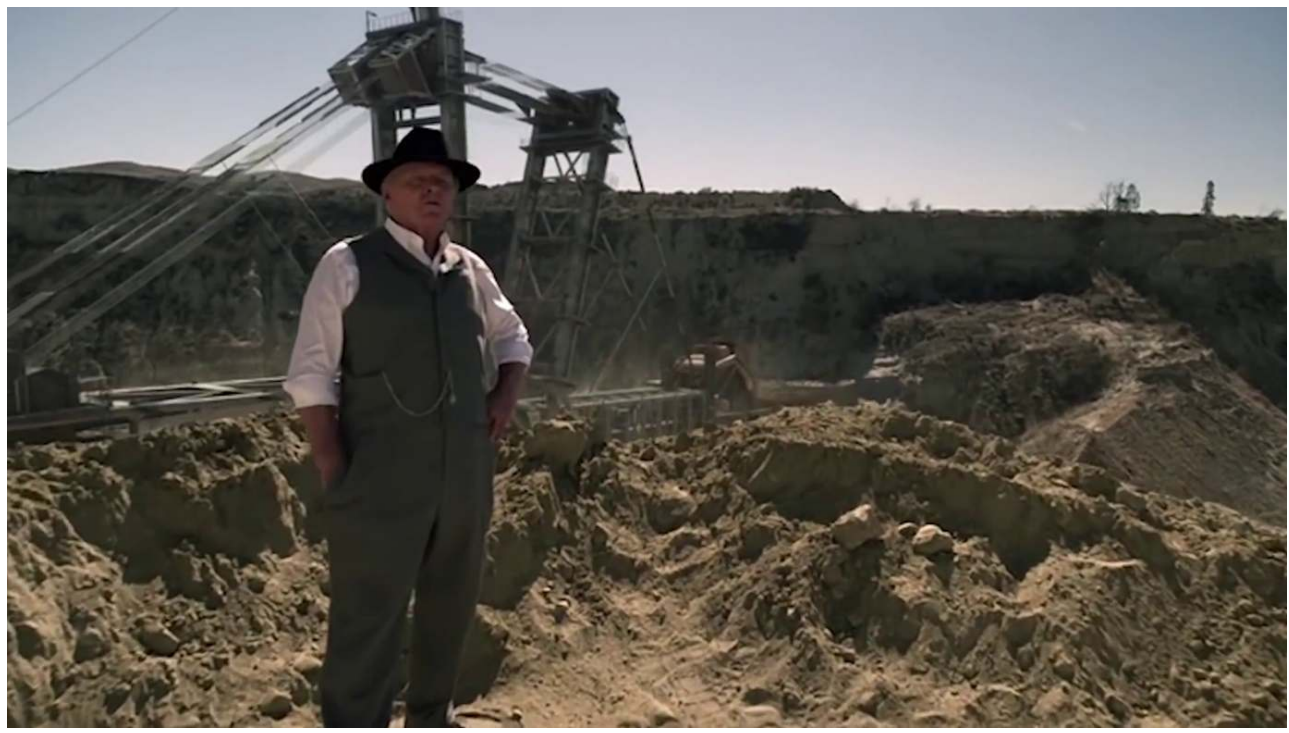

Fig. 4 : Excavation : le réel palimpseste

La fouille archéologique d'un réel palimpseste qu'incarne ici Ford est en tout point conforme à celle qu'opère Voss dans sa vidéo. On trouve par ailleurs un grand nombre de vidéos similaires sur Internet, comme celle qui reprend « tous les signes que Bernard était en fait Arnold que vous avez laissé passer quand vous avez regardé la série ${ }^{29} »$. Le 
principal indice est bien sûr que le nom de l'un est l'anagramme de celui de l'autre, interprétation cabalistique classique que de nombreux spectateurs avaient très tôt été capables de produire (Fig. 5). Mais, montrant un certain sens de l'auto-ironie, Voss se reproche d'avoir été parmi les derniers à identifier cet indice grossier. Ce qu'il décrit, c'est en quelque sorte le syndrome de La Lettre volée d'Edgar Allan Poe $^{30}$, mais adapté à l'ère digitale: on n'oublie plus d'inclure dans sa recherche ce qui saute aux yeux, mais une opération de décodage anagrammatique considérée comme un cliché du genre et rangée, de ce fait, dans l'ordre de l'évidence.

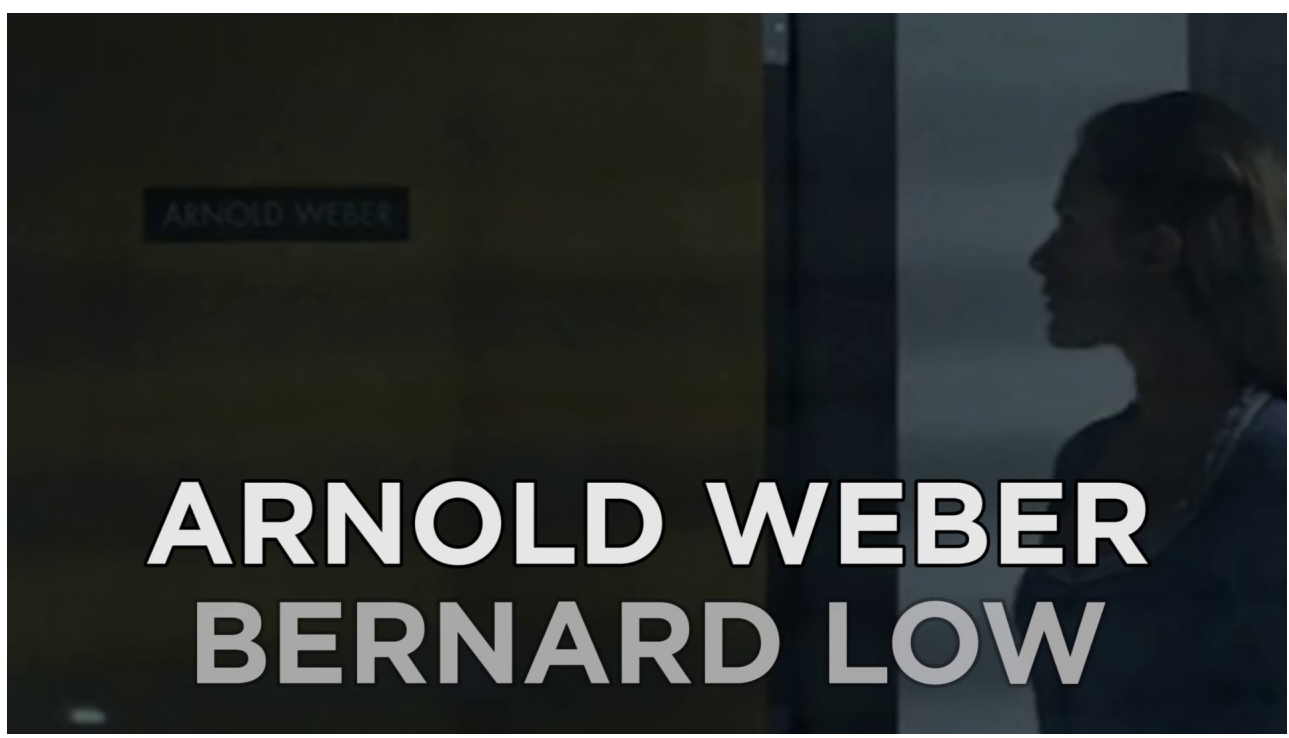

Fig. 5 : Logique anagrammatique

17 La vidéo de Voss, comme toutes celles de ce type, résulte d'un processus cognitif qui consiste à récolter, trier, et interpréter les informations en fonction d'une théorie (ici à rebours, en utilisant les révélations finales comme point de départ $\left.{ }^{31}\right)$. Le but est de ramener des éléments disparates à un tout cohérent, dont l'unité renforce le pouvoir de conviction de la théorie développée. Néanmoins, comme le résultat peut paraître relever de la surinterprétation, il n'est pas inutile de répéter le raisonnement dans une version encore simplifiée, comme Voss le fait ici en énumérant les diverses étapes du plan secret mis en œuvre par Ford. Il indique ensuite avoir suivi, dans une autre de ses vidéos, le même type de raisonnement hypothético-déductif à propos de la théorie des temporalités multiples, apparue suite à l'interprétation par les spectateurs d'indices décisifs, trouvés notamment dans l'épisode 7. La probabilité identifiée qu'il existe des temporalités multiples est devenue probable plutôt que possible à mesure que les scénaristes disséminaient des indices pour alimenter la "méthode du détective ${ }^{32}$ " qu'utilisent les spectateurs doublés d'internautes, en parfaite adéquation avec la croyance complotiste selon laquelle tous les événements ont forcément un lien entre eux, et ne prennent sens que si on les rattache à un plan d'action de grande ampleur, totalitaire et secret ${ }^{33}$.

18 Mais selon la lecture complotiste, le passé ne fait pas que produire le présent : il permet également d'entrevoir l'avenir, comme les fouilles d'Escalante annoncent l'état de futur achèvement du parc - et dévoilent en filigrane le plan de Ford ${ }^{34}$. Cette croyance apocalyptique motive ici un certain nombre de prédictions pour la suite de la série, concernant Ford notamment. Les conjectures de Voss, comme souvent dans les récits complotistes, ont par ailleurs recours à une causalité étayée par des motifs psychologiques $^{35}$. Pour conjurer le vieillissement, Ford se serait lui-même cloné en hôte. 
Ces motifs psychologiques suggèrent que la fin de la première saison ne suffit pas en soi. La découverte de la nature des agissements de Ford n'exclut pas que son plan soit, en fait, toujours en train de se dérouler plutôt qu'achevé en fin de première partie.

19 Car une fois découvert, un complot peut toujours en révéler un autre à plus grande échelle ${ }^{36}$, comme par exemple, ici, s'il s'avérait que Ford, malgré sa mort théâtrale, n'avait pas renoncé à dominer Westworld, mais qu'il comptait continuer à exercer son contrôle par robot interposés ${ }^{37}$. Ainsi, Ford représente cette peur des dérives de la science et d'une technologie sans frein qui caractérise la culture complotiste selon Gulyas ${ }^{38}$. Il incarne également en cela le « grand horloger » qui, dans la culture paranoïaque, fait renaître une figure d'autorité disparue en même temps que la confiance des humains dans le réel ${ }^{39}$. Comme pour toutes les théories du complot, celle d'un Ford qui se serait lui-même cloné est en concurrence avec des versions alternatives ${ }^{40}$, que Voss envisage lui-même. Il estime possible que son raisonnement soit tiré par les cheveux, pour évoquer ensuite un autre élément venant à l'appui de sa théorie : Antony Hopkins a tweeté qu'il serait présent dans la saison suivante.

Par conséquent, comme le montre ici la valse-hésitation de Voss, la réception complotiste n'est jamais satisfaite. Aucune révélation ne saurait être concluante : elle ferait dans ce cas naître la frustration de l'impossibilité d'une suite. Or, à l'image de l'homme en noir qui en est le vecteur, le sentiment qui doit prévaloir après la première saison est la certitude et la nécessité d'une deuxième où seront élucidés d'autres mystères. L'homme en noir évoque cet aspect lorsqu'il décrit sa relation addictive au parc: "C'est ça que j'aime bien dans cet endroit, tous les secrets, toutes les petites choses que je n'ai pas encore remarquées, malgré toutes ces années. Tu sais pourquoi c'est mieux que le monde réel, Lawrence ? Parce que le réel est chaotique. Le réel est un accident. Mais ici, chaque détail est signifiant ${ }^{41}$. » Il n'est donc guère étonnant que, poussés de la sorte à poursuivre leur quête d'indices, de nombreux internautes aient pesé le pour et le contre de l'épisode 10 , alors que d'autres se sont de suite emparés de la bande-annonce de la deuxième saison pour en tirer de nouvelles théories.

21 Enfin, le stade ultime de la théorie complotiste, qui est censé en cimenter la crédibilité, consiste à proposer une vision du monde schématique, censée être plus claire et accessible à tous. Pour cela, on peut présenter la carte mentale du raisonnement tenu pour qu'il semble imparable ${ }^{42}$. Dans Westworld, le schéma explicatif ainsi livré aux spectateurs est bien sûr celui du labyrinthe, qui figure partout dans la série. Il en existe d'autres, comme la pyramide de la conscience, qui, selon Voss explique le dessein de Ford, et cartographie l'architecture de la première saison au niveau narratif (Fig. 6) ${ }^{43}$. 


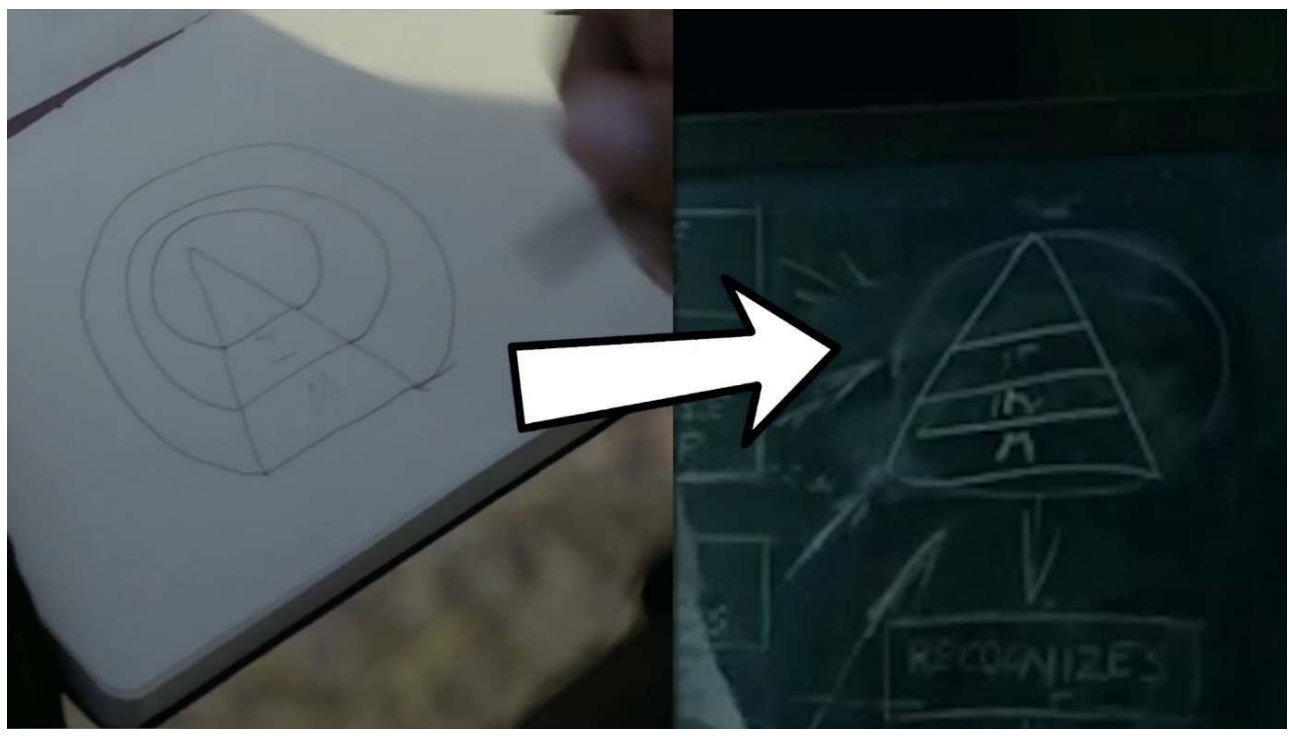

Fig. 6 : Parcours fléché 2 : la pyramide de la conscience

22 Ce travail de cartographie, également caractéristique de la réception complotiste, peut dans certains cas être plus totalisant, comme le montrent les diverses cartes que l'on trouve sur Internet, et qui sont destinées à aider les spectateurs à se repérer dans ce gigantesque «parc narratif » qu'est la série (Fig. 7) ${ }^{44}$.

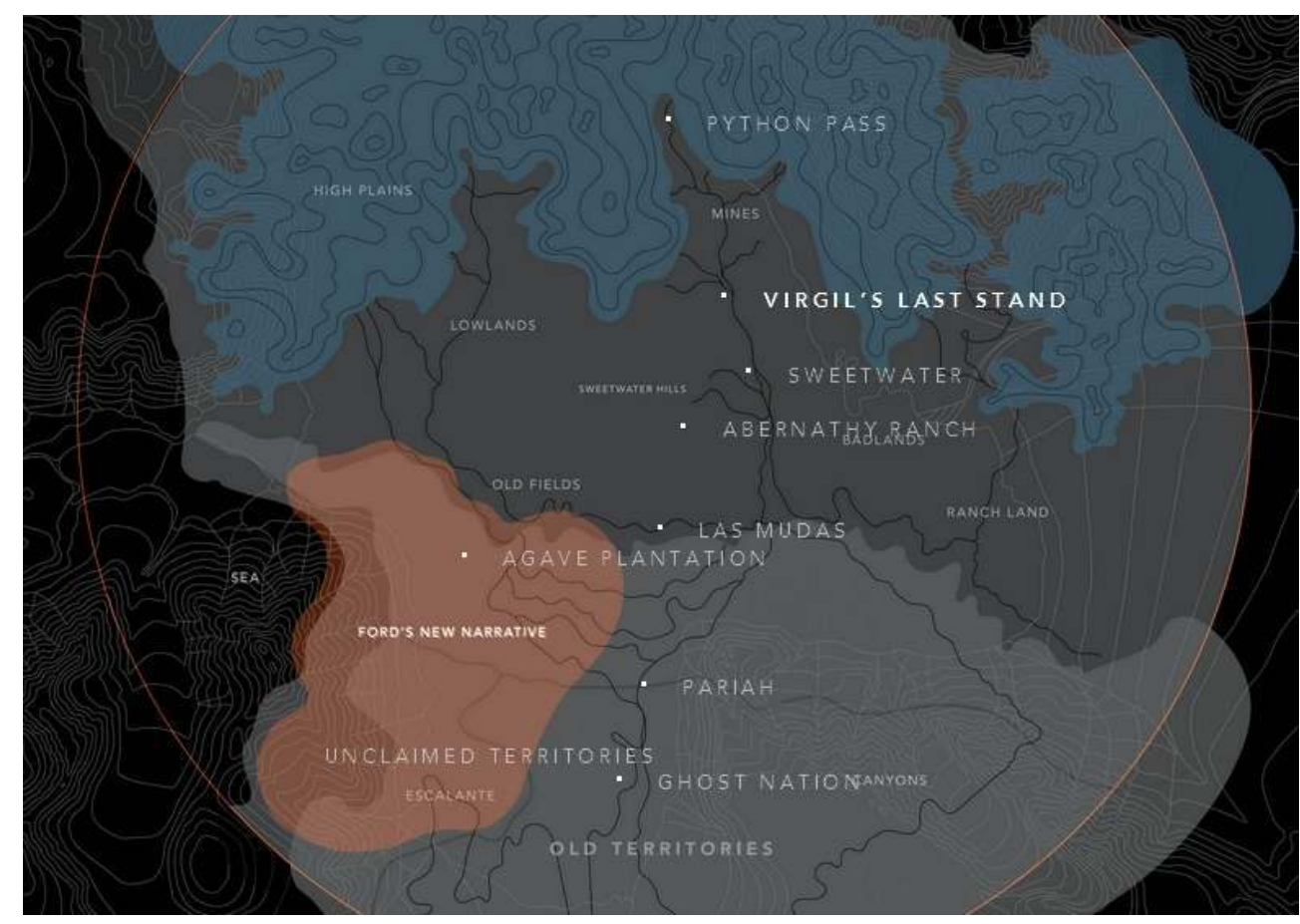

Fig. 7 : Parcours fléché 3 : cartographie et orientation

23 Après avoir évoqué la pyramide, Voss propose un commentaire du passage où Ford analyse une œuvre connue, La Création d'Adam de Michel-Ange (Fig. 8). Il l'utilise cependant comme une énième invitation à regarder attentivement pour voir ce qui est " caché dans le visible » (hidden in plain sight) - à la différence près qu'il ne parle plus ici du monde de la série, mais d'une fresque bien réelle ${ }^{45}$. 


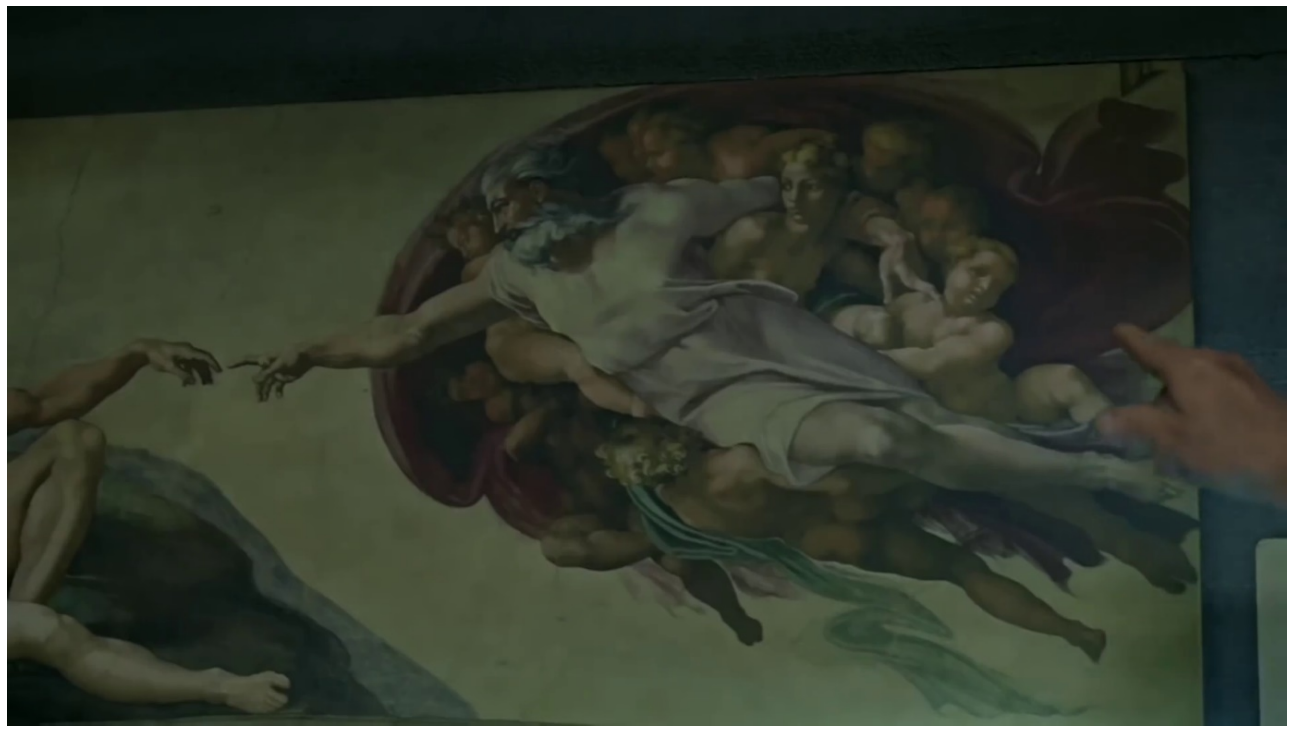

Fig. 8 : Parcours fléché 4 : décryptage esthétique du réel

La paranoïa contemporaine permet d'échapper au quotidien, en se réfugiant dans un récit addictif, comme le fait le vidéaste. Elle est source de plaisir, notamment par son aspect auto référentiel et distanciant, que caractérise le mode ironique de sa posture ${ }^{46}$. En conséquence de quoi, la paranoïa apparaît comme le dernier refuge identitaire dans un monde parodique, où tout ne serait que simulacre ${ }^{47}$ : Voss joue avec sa propre identité et utilise l'autodérision pour souligner que sa lecture est déniaisée, et qu'il n'ignore pas que la valeur de la série est avant tout métaphorique (il emploie le terme).

Mais après un début très convaincant, l'explication psychologique de Voss devient de plus en plus complexe. Elle aboutit néanmoins, comme l'exige le genre, à une théorie simple, confirmée par la série dans son dernier épisode. L'accès à la conscience des robots n'est pas réel : il est le fruit d'un programme implanté par Ford, qui transforme Dolores en Wyatt, et la voix qu'elle entend n'est pas la sienne uniquement, mais bien celle d'Arnold superposée à celle de Ford. On comprend donc que la bicaméralité évoquée dans le titre du dernier épisode (The Bicameral Mind) fait ici l'objet d'un détournement. Théorie psychologique, elle devient un simple code, un programme informatique implanté dans un robot, une série d'informations numériques pouvant se réduire à des 1 et à des 0 . Cette ultime révélation rappelle que pour de nombreux auteurs, la paranoïa actuelle est l'ultime stade épistémologique, avant la transformation de la totalité du savoir humain en simple information ${ }^{48}$. Un autre signe de cette reductio ad numericum du savoir humain intervient peu après dans la vidéo, au moment où Voss informe les spectateurs qu'un personnage dont le sort est resté en suspens lors de la première saison, Elsie, peut être géolocalisée sur le site Internet de Delos (Fig. 9). Cependant, l'accès à cette révélation passe obligatoirement par la lecture d'un code informatique ${ }^{49}$, ce qui constitue une invitation lancée au public à participer au récit, en décodant un univers narratif. Cet impératif est en parfaite conformité avec une tendance en place chez les publics de la culture populaire depuis au moins le milieu des années quatre-vingts et le concept d'œuvre ouverte tel que théorisé par Umberto $\mathrm{Eco}^{50}$. 


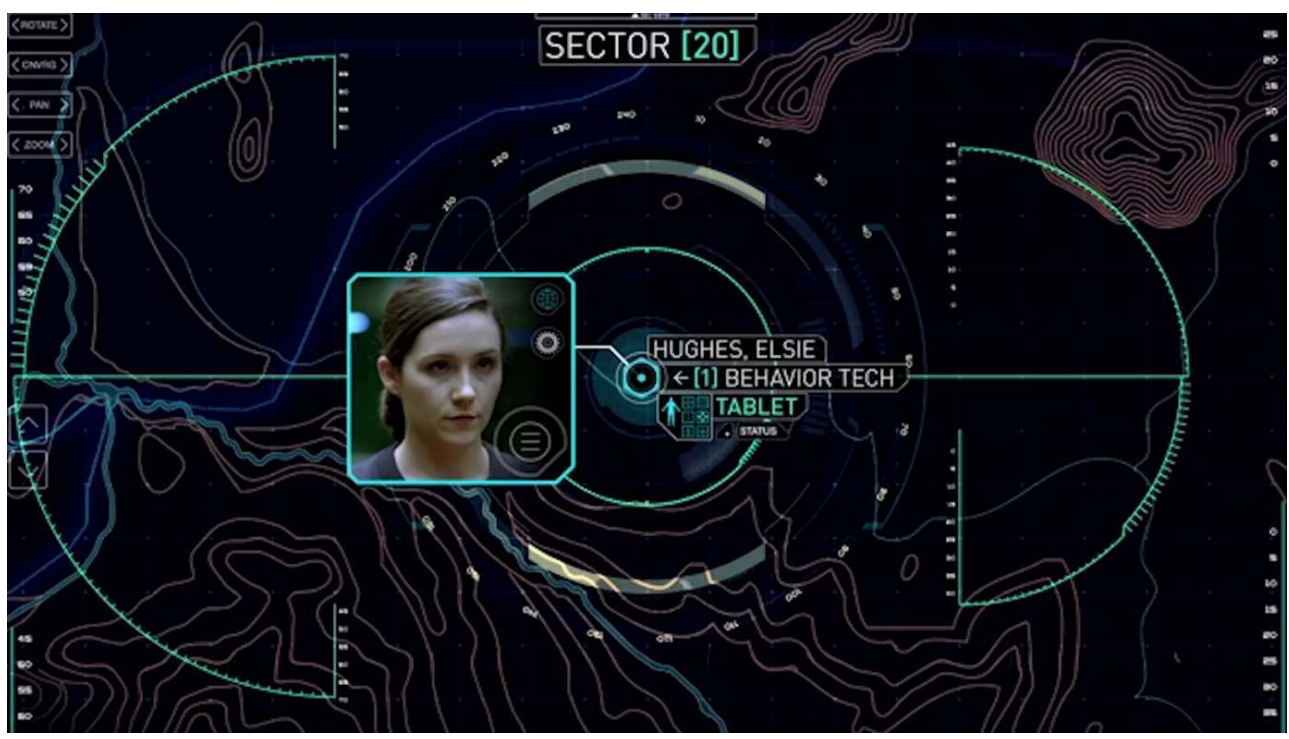

Fig. 9 : Parcours fléché 5 : où est Elsie?

\section{Westworld, vecteur du complotisme?}

La vidéo de Voss montre donc, dans les passages où elle évoque un décodage de l'œuvre de Michel-Ange qui apparente Westworld au Da Vinci Code, ou quand elle met en évidence une piste à suivre sur Internet, à quel point la série utilise des techniques qui sont souvent sources de paranoïa. Sa particularité est de le faire à la fois en deçà et au-delà des limites de son univers diégétique. On est dès lors en droit de se demander si Westworld n'est pas elle-même un vecteur de développement du complotisme par-delà les limites de son univers fictionnel.

Pour que cette hypothèse soit valable, il est nécessaire, d'une part, que Westworld mobilise les caractéristiques dont on considère qu'elles sont susceptibles d'alimenter le complotisme. Ces « vecteurs du complotisme » sont les causes les plus souvent évoquées pour expliquer la popularité actuelle des théories du complot : l'instabilité économique et géopolitique $^{51}$, la surinformation ${ }^{52}$, et la perte de confiance en l'autorité des intellectuels et des experts qui l'accompagne ${ }^{53}$, une peur panique de la perte de contrôle sur sa destinée et un sentiment généralisé d'impuissance ${ }^{54}$, la surveillance constante et ubiquitaire ${ }^{55}$, et enfin, une crise conjoncturelle liée à la condition postmoderne ${ }^{56}$. Par ailleurs, pour que ce raisonnement soit valable, il faut également que ces vecteurs $d u$ conspirationnisme montrent une certaine propension à dériver du fictionnel vers le réel. L'exemple de Michel-Ange, comme celui d'Elsie, relèvent déjà d'une telle tendance. En fondant ces deux hypothèses, on pourra supposer que Westworld met en scène dans un cadre fictionnel des situations favorisant le conspirationnisme, mais que les conséquences de ces situations sont parfois bien réelles.

Le premier élément de réponse à ce questionnement tient de l'évidence. Westworld s'appuie, par son dispositif narratif-même, où les robots sont indiscernables des humains, sur le cliché postmoderne d'une confusion entre le réel et le fictionnel, qui suggère que s'est opérée une fusion entre ces deux notions. Il s'agit également d'un trait caractéristique de la culture du complot, qui laisse à penser que le réel est la mise en scène d'un récit maitrisé de part en part, uniquement accessible à un cercle restreint 
d'initiés capables de dépasser la tromperie des apparences et de lire entre les lignes. Mais la série va plus loin, puisqu'elle cultive cette confusion. Elle en inverse même le sens habituel, en encourageant une perception du réel comme palimpseste à décrypter afin d'en percer le véritable sens et d'en déceler le fonctionnement caché. Les modalités typiques du postmodernisme qui facilitent un transfert complotiste du récit vers le réel sont nombreuses dans Westworld. Les premières concernent la paranoïa précédemment évoquée. La paranoïa actuelle est souvent considérée comme la seule réaction possible au capitalisme qui bouleverse le matériel comme le temporel ${ }^{57}$. Or, la vidéo de Voss identifie une certaine marchandisation de Shakespeare (à travers l'utilisation simpliste de ses répliques, mais également à travers des adaptations grand public comme le Romeo + Juliet de Luhrmann, dont on voit quelques images ${ }^{58}$ ). Elle évoque aussi une exploitation mercantile du Westworld de Crichton sorti en 1973 (dont le contenu change de manière assez radicale, car seule l'idée de base est reprise). Voss évoque par ailleurs l'influence de Westworld sur sa propre lecture du réel, lorsqu'il déclare, à propos d'un collègue YouTubeur, «He is like the Ford to my Bernard ». Enfin, il établit le lien entre l'entreprise Delos, qui appartient à l'univers de la série, et son site Internet, qui se présente comme un site Internet classique, ce qui entretient le doute sur l'existence réelle ou non de Westworld - le parc e(s)t la série ${ }^{59}$.

Cette dernière s'inscrit donc dans un univers transmédiatique complémentaire et englobant qui en multiplie les profits. On peut se contenter d'y voir une stratégie marketing, une sorte de placement de produit culturel multi-support que symboliserait le logo du parc, qui est également le logo de la série. On pense en effet au Frontierland de Disney : à quand un méta-parc dont les visiteurs seraient plongés dans l'univers non pas du western, mais de Westworld? Néanmoins, certains internautes accordent plus d'importance à ce phénomène, en évoquant, à partir d'une lecture de Westworld, la possibilité que le monde actuel soit gouverné en secret par les forces capitalistes - ce qui est loin d'être un raisonnement original, sauf à considérer que les forces en question agissent de manière indécelable et quasiment subliminale, comme le pensent les adeptes de théories du complot. C'est ce que l'on trouve sur une page de forum, semblable à tant d'autres, où l'un des intervenants déclare : «[...] dans Westworld, c'est comme dans la vie. Tout est fait pour que les riches s'en mettent plein les poches ${ }^{60} »$. Mais Westworld n'est pas la seule série à être concernée par cette théorie d'un complot capitaliste. Dans un article de juillet 2017, le site du magazine Première relayait les propos d'un présentateur radio, Alex Baker, qui impose la même théorie à Friends : la sitcom serait un moyen subliminal trouvé par Starbucks pour « faire sa pub » et inciter les gens à aller boire du café6 ${ }^{61}$.

Toujours selon la même logique d'enchaînement des opérations cognitives, cette théorie peut aller toujours plus loin, en considérant comme un même ensemble plusieurs séries de HBO, dont les univers narratifs auraient des zones de recoupement, et dont Westworld ne serait qu'un chaînon. On peut le voir dans une autre théorie, selon laquelle le Westeros de Game of Thrones serait en fait Westworld, ce qui suggère que les univers narratifs sont liés entre eux par l'industrie médiatique, qui encourage des rapprochements fantasques afin que la consommation d'une série mène à celle d'une autre, puis à une autre, et ainsi de suite $^{62}$. À lire ces théories, on soupçonnerait presque l'existence d'un complexe narrativo-industriel.

31 Ce type de raisonnement révèle surtout une perte de confiance en l'autorité des auteurs, qui seraient privés de tout contrôle artistique par les cadres de l'entreprise qui les emploie, à l'instar de Sizemore, le scénariste impuissant, et finalement déchu, du parc. De 
fait, les raisonnements tenus par certains internautes entretiennent ce sentiment: apparemment, les théories qui circulent sur la deuxième saison, basées sur une interprétation de la bande-annonce, auraient conduit les scénaristes à changer d'intrigue ${ }^{63}$.

On peut par ailleurs mettre ce rapprochement en rapport avec une autre cause communément évoquée de la popularité du complot: l'hyper-accessibilité de l'information suscitant une perte de confiance en l'autorité des intellectuels et des experts. L'exemple du parallèle phonético-sémantique entre Westeros et Westworld n'est pas le seul que l'on puisse opérer, à titre individuel, en allant chercher des informations sur Internet pour étayer un raisonnement. On peut en imaginer d'autres, autant par jeu que pour illustrer par l'exemple le fonctionnement de la « raison complotiste ». Dans la vidéo de Voss, on trouve un arrêt sur l'image du moment où Bernard pointe un pistolet sur son crâne (Fig. 10). Le commentaire de Voss remarque que l'angle de tir s'écarte volontairement de l'horizontale, afin que le cerveau de Bernard ne soit pas complètement détruit.

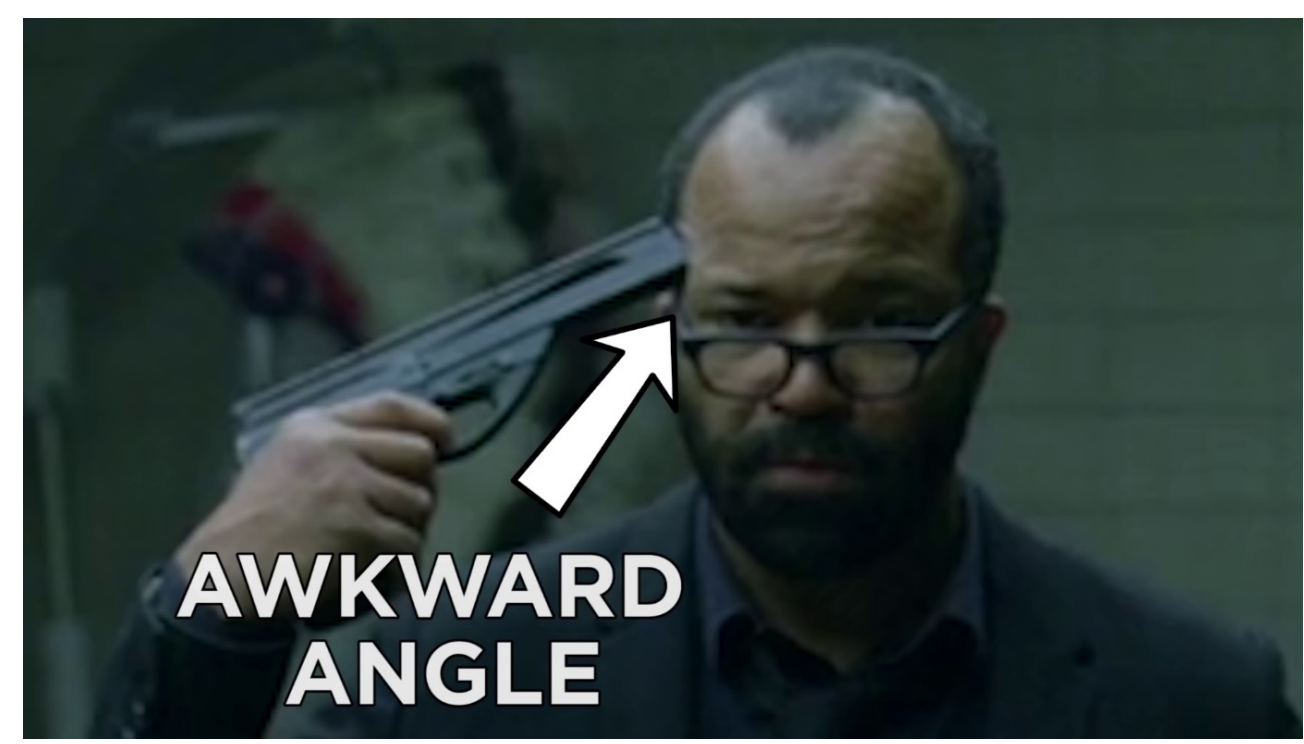

Fig. 10 : Parcours fléché 6 : logique balistique

Il est tentant de rapprocher cette interprétation d'une autre théorie, bien réelle celle-là : la théorie de l'expert en balistique Howard Donahue sur l'assassinat de Kennedy, qui s'appuie sur une analyse de la trajectoire de la balle et de la nature de la blessure provoquée par cette dernière pour expliquer que Kennedy a en fait été tué accidentellement, par la maladresse d'un agent des services secrets chargé de le protéger ${ }^{64}$. Bien sûr, cette théorie révèle une absence de complot, mais elle s'oppose à d'autres théories balistiques prouvant que l'assassinat de Kennedy est le fruit d'une conspiration, à commencer par celles qui critiquent les incohérences de la théorie dite « de la balle unique ", défendue par la commission Warren. Quoi qu'il en soit, il est évident que Voss utilise la balistique dans un cadre complotiste, ce qui n'est guère étonnant puisque, comme le rappelle Fenster, la théorie du complot est devenue un trait prédominant de la culture politique mondiale, après l'assassinat de Kennedy en $1963^{65}$. 


\section{Conclusion}

34 Je terminerai d'élaboration de mon hypothèse selon laquelle le complotisme de Westworld aide les théories du complot à prospérer par une étonnante illustration, trouvée sur le site du Guardian. L'article, paru le 30 octobre 2016, c'est-à-dire alors que la saison 1 arrivait à la fin de sa première moitié, recense les théories les plus folles sur la série ${ }^{66}$. Les premières ont été confirmées depuis : Bernard est un robot, l'homme en noir est un milliardaire, et Westworld recourt à des temporalités multiples. Néanmoins, une hypothèse plus fantasque figure également dans l'article : tous les personnages sont peutêtre des robots, ce qui encourage un doute méthodique sur la nature du réel, signe d'une crise d'identité conjoncturelle liée à la postmodernité.

La suite est encore plus surprenante. Westworld serait une gigantesque expérience de psychologie comportementale, semblable à l'expérience de Stanford ou à celle de Milgram. On est passé du sentiment d'être observé en permanence à la conviction de servir de cobayes, vivant dans une réalité factice qui nous cache la réalité de notre condition. D'où la théorie numéro six, qui mobilise le cliché postmoderne du simulacre, dont Jagodzinski nous rappelle à quel point il est compatible avec la sérialité : la dimension répétitive ancre l'idée d'un univers où tout ne serait que feintise ${ }^{67}$. Selon cette théorie, tout dans Westworld se passe comme dans Matrix, le monde de la série est entièrement virtuel, et rien n'y existe réellement. Il s'agit d'une reprise quasiment terme à terme de la théorie du simulacre, où la simulation apparaît comme un système de signes devenu suffisamment autonome et sophistiqué pour abolir son propre référent et en tenir soi-même lieu ${ }^{68}$. En effet, du point de vue des robots, le parc est une simulation parfaite, un décor en trompe-l'œil dont ils vont petit à petit découvrir l'envers, suivis en cela par les téléspectateurs et téléspectatrices soucieux et soucieuses de percer cette illusion à jour. Mais ne nous y trompons pas. Ce n'est pas de nous que parle l'article, mais des personnages de la série. À moins que, comme le défend la théorie numéro sept, Westworld ne soit en vérité un documentaire terrifiant sur notre condition. Cette dernière théorie repose sur les récents propos d'Elon Musk, dont on connaît la paranoïa par rapport aux progrès réalisés par l'intelligence artificielle. Selon certaines de ses théories, qui évoquent l'existence d'un complot transhumaniste, il serait assez vraisemblable que nous vivions d'ores et déjà au sein d'un simulacre. Westworld révélerait alors notre vérité, en documentant notre condition de manière réflexive, pour nous montrer que nous vivons une illusion, et nous aider à en sortir, comme Maeve.

Une telle lecture fournit la dernière pièce de notre puzzle, le principe fondateur du conspirationnisme que la série ne nous avait pas encore, jusqu'ici, permis d'évoquer. Selon ce principe, « le complot n'est pas une 'théorie'. De fait, il est partout, pour ceux qui sont capables de regarder attentivement et de décoder le monde qui les entoure ${ }^{69}$. » Qu'on ait implanté à notre insu une puce électronique dans notre crâne, ou qu'on ait placé une idée fixe dans nos méninges suite à un lavage de cerveau, il est certain que son rôle, plutôt que de susciter un simulacre de réel, est d'inciter à un décodage permanent ${ }^{70}$, où l'on peut voir la dernière itération en date d'un phénomène ancestral : le brouillage des frontières entre le réel et la fiction ${ }^{71}$. Cependant, l'aspect sous lequel ce brouillage nous apparaît ici pose question par son renversement de l'ordre habituel où le réel rejoint, voire dépasse, la fiction. Car dans le cadre du conspirationnisme post-humain, dont on trouve ici un bel exemple, c'est bien une fiction comme Westworld qui sert de substrat à la 
construction d'une hypothèse réaliste. L'inverse, en somme, de ce que propose Black Mirror, et que j'ai appelé par ailleurs une " dystopie intégrée ${ }^{72}$ ", où l'implant qui décuple les facultés humaines symbolise des technologies d'ores et déjà disponibles. Westworld, $a$ contrario, est un exosquelette fictionnel, porteur d'un complotisme post-humain auquel la série donne corps et crédibilité, au risque d'en faciliter l'avènement.

\section{NOTES}

1. Le présent travail porte exclusivement sur la première saison de la série, diffusée du 2 octobre au 4 décembre 2016, et par conséquent seule en date au moment de la rédaction de cet article. Il me paraît néanmoins utile de préciser que la 2e saison, qui vient d'être diffusée entre avril et juin 2018, confirme en de nombreux points la lecture qui est proposée ici. Comme elle fera l'objet d'un autre article, où sera exploré le lien entre surveillance panoptique du parc, création d'un data double, et dénonciation des mirages post-humains, je fais le choix de ne pas y faire référence de manière plus précise pour l'instant, bien que l'ayant visionnée avec le plus grand intérêt.

2. Mark Fenster, Conspiracy Theories: Secrecy and Power in American Culture, Minneapolis, University of Minnesota Press, 2008, p. 1. Ma traduction.

3. En tant que récit complotiste, Westworld possède également une parenté évidente avec les trompe-l'œil cinématographiques analysés par Aurélie Ledoux dans L'ombre d'un doute, le cinéma américain et ses trompe-l'œil, films dont les pièges perceptifs constituent autant d'invitations à penser que le réel n'est qu'un écran de fumée. Aurélie Ledoux, L'ombre d'un doute : le cinéma américain contemporain et ses trompe-l'oeil, Rennes, Presses Universitaires de Rennes, 2012.

4. Certains, à l'image du bloggeur Peter Sciretta, ont d'ailleurs repéré très vite cette tendance, allant jusqu'à tenir un listing des théories les plus en vogue. «After the first episode of Westworld aired, we became inundated with fan theories. Some of the Westworld theories were rather unlikely, others possibly mind-blowing. Showrunner Jonathan Nolan has even debunked a few of them. Well now that Episode 2 has aired, there are a whole batch of new Westworld theories, some of them just as crazy and intriguing as the others we've posted. »

5. Sur cette question, on peut se référer à l'article fondateur de Richard Hofstadter, "The Paranoid Style in American Politics ", Harper's Magazine, novembre 1964, p. 1, https:// harpers.org/archive/1964/11/the-paranoid-style-in-american-politics/5/.

6. Il s'agit en particulier de montrer que les citations shakespeariennes sont autant d'invitations à l'interprétation sur le mode du message codé, le réseau de répliques extraites des pièces constituant un filigrane censé aider le public à percer le mystère de la série. Westworld n'est bien entendu pas la seule série à adopter ce fonctionnement. Selon Jagodzinski, un grand nombre de séries télévisées font de la réflexivité des dialogues un remède à l'effondrement apocalyptique de l'univers symbolique. Jan Jagodzinski, Television and Youth Culture, Basingstoke, Palgrave Macmillan, 2009, p. 21. Les citations de Shakespeare jouent un rôle similaire dans Westworld.

7. Mon propos dans cet article consiste également à montrer, plus systématiquement qu'ici, que ces répliques incitent les spectateurs et spectatrices à un décodage collaboratif s'effectuant notamment sur Internet. Sur le lien entre culture de la paranoïa et autoréflexivité, on peut également se rapporter à Patrick O'Donnell, Latent Destinies: Cultural Paranoia and Contemporary U.S. Narrative, 2000, p. 9.

8. Mark Fenster, Conspiracy Theories, op. cit., p. 6. 
9. Julia Alexander, « Westworld is bigger than The Walking Dead, Game of Thrones in its first season ", Polygon, 15 novembre 2016, consulté le 4 février 2018, https://www.polygon.com/ tv/2016/11/15/13640916/westworld-game-of-thrones-walking-dead-ratings.

10. Mark Fenster, Conspiracy Theories, op. cit., p. 125.

11. Ibid., p. 142.

12. Ibid., p. viii.

13. «This is it? The center of the maze?»

14. «It ends with a place I've never been, a thing I'll never do ».

15. THE MAN IN BLACK. How am I doin' Robert? Any closer to what I'm lookin' for?

FORD. I don't know. What is that? »

16. " Have you ever questioned the nature of your reality? »

17. Michael Butter, Plots, Designs, and Schemes: American Conspiracy Theories from the Puritans to the Present, de Gruyter, 2014, p. 44.

18. "Figuring out how it works is half the fun ".

19. Jan Jagodzinski, Television and Youth Culture, op. cit., p. 6.

20. Erik Voss, Westworld Ending Explained (Ford's Master Plan, The Maze \& Dolores), https:// www.youtube.com/watch? $\mathrm{v}=\mathrm{K} 9 \mathrm{AwvWGjJeQ}$. J'ai bien conscience des limites que peut présenter une étude portant sur un seul exemple de réception. Néanmoins, comme cette vidéo qui décrypte la série selon un mode de réception complotiste a été visionnée 2339176 fois à la date d'écriture du présent article, on peut extrapoler sur la représentativité de son contenu et supposer qu'une telle réception est partagée et approuvée par plusieurs millions de spectateurs. Ce nombre sera d'autant plus significatif qu'on le comparera au nombre de spectateurs de chaque épisode de Westworld (entre 2 et 3 millions selon les estimations). Enfin, on peut également préciser, à l'appui de cette démarche, qu'il existe un grand nombre de vidéos du même type sur Internet, comptant chacune un nombre important de vues.

21. Jan Jagodzinski, Television and Youth Culture, op. cit., p. 6.

22. «I made another video breaking down the convoluted timeline of Westworld reordering the story in the exact chronology [...] But in this video I'm gonna quickly explain what the hell we just saw because let's be honest, Westworld threw a lot at us at once ». (Voss, op.cit.)

23. « This season finale revealed way more than I ever expected from a show produced by J. J. Abrams ». (Voss, op. cit.)

24. Voir Robert M. Fowler, Let the Reader Understand: Reader-Response Criticism and the Gospel of Ma rk, A\&C Black, 2001, p. 36-37.

25. "Conspiracy beliefs are associated with mental illness, including paranoia, obsession, psychosis ... with being antisocial, including crackpots and despicable and bigoted people. » Lance deHaven-Smith, Conspiracy Theory in America, University of Texas Press, 2013, p. 129.

26. «That was the sound of thousands of conspiracy theorists climaxing all at once ». (Voss, op. cit.)

27. Cette reconstitution à partir de détails est typique du comportement des internautes. " Multiple versions of the same document are likely to appear in various places, some identical, some slightly different, some with annotations by the poster. (...) All of the variants may in principle be simultaneously available to the web surfer (...). Here repetitions substitutes for direct evidence as a way of determining veracity. " Michael Barkun, A Culture of Conspiracy: Apocalyptic Visions in Contemporary America, Berkeley, University of California Press, 2013, p. 13.

28. "So all season long we saw Ford preparing his new secret narrative, digging up the city of Escalante and repurposing hundreds of hosts ». (Voss, op.cit.)

29. Terri Schwartz, Every Time Westworld Hinted at the Big Reveal - IGN Video, consulté le 4 février 2018, http://www.ign.com/videos/2016/11/14/every-time-westworld-hinted-at-the-bigbernard-reveal. 
30. Rappelons que, dans la nouvelle, la lettre recherchée reste très longtemps introuvable d'avoir été cachée aux yeux de tous, et que la « lettre volée » représente l'idée que ce qui est trop visible n'attire paradoxalement que rarement l'attention, en particulier lorsqu'une quête est en cours.

31. Mark Fenster, Conspiracy Theories, op. cit., p. 125.

32. Voir Matthew R. X. Dentith, "Conspiracy Theories and Their Investigator(s) ", Social Epistemology Review and Reply Collective 6, no. 4 (2017): 4-11, p. 5, consulté le 19 février 2018, https://social-epistemology.com/2017/04/13/conspiracy-theories-and-their-investigatorsmatthew-r-x-dentith/.

33. Richard Hofstadter, « Paranoid Style ", op. cit., p. 84.

34. Patrick O'Donnell, Latent Destinies, op. cit., p. 9.

35. David Bordwell, Janet Staiger et Kristin Thompson, The Classical Hollywood Cine ma, London, Routledge, 2006, p. 12.

36. Comme le rappelle Giles Scott-Smith, c'était déjà une telle théorie des " complots gigognes » qu'employait George Kennan à propos du gouvernement soviétique en 1946. Giles Scott-Smith, Western Anti-Communism and The Interdoc Network, Basingstokes, Palgrave MacMillan, 2012, p. 1.

37. "Some people are thinking that Ford may also be hinting that he built a host version of himself, that this Ford may just be a host or this is the real Ford and there's another host version of him somewhere that's ready to carry on his legacy ». (Voss, op.cit.)

38. Aaron John Gulyas, Conspiracy Theories: The Roots, Themes and Propagation of Paranoid Political and Cultural Narratives, Jefferson, NC, McFarland, 2016, p. 17.

39. Jan Jagodzinski, Television and Youth Culture, op. cit., p. 7.

40. Voir Mark Fenster, Conspiracy Theories, op. cit., p. 94.

41. "That's what I love about this place, all the secrets, all the little things I never noticed even after all these years. You know why this beats the real world, Lawrence? The real world is just chaos. It's an accident. But in here, every detail adds up to something. »

42. Sur le lien entre complot et cartographie, voir Fran Mason, «A Poor Person's Cognitive Mapping ", in Peter Knight (dir.), Conspiracy Nation: The Politics of Paranoia in Postwar America, New York, New York University Press, pp. 40-56, p. 43-44. « Narrative provides a form of mapping for conspiracy theory, offering not only an explanatory history but also a map of the future that is to come.»

43. " Initially Arnold imagined this journey as a pyramid with different layers, the bottom one being memory because humans need to remember things to learn from them and then improvisation because humans tend to deviate from their routine. » (Voss, op.cit.)

44. "The Map of the "Westworld" Park Facility Shows Where Everything Is ", Inverse, consulté le 4 février 2018, https://www.inverse.com/article/22284-westworld-delos-map-mesa-gold.

45. " Notice how Ford repeated that idea later when describing the brain shape hidden in Michelangelo's painting. FORD. 'It took 500 years for someone to notice something hidden in plain sight “» (Voss, op. cit.)

46. "If you've never suffered you're not human, which is a huge relief because all I do is suffer. » (Voss, op. cit.)

47. Patrick O'Donnell, Latent Destinies, op. cit., p. 9.

48. Paranoia is «the last epistemological, the final form of human knowledge before it passes away into information ». Ibid.

49. « [Voss:] Delos corporation posted a mysterious code that when you decode it shows Elsie's coordinates in the park and an audio file that sounds a lot like her voice. »

50. Jan Jagodzinski, Television and Youth Culture, op. cit., p. 61.

51. Martha F. Lee, Conspiracy Rising: Conspiracy Thinking and American Public Life, ABCCLIO, 2011, p. 72-73.

52. Ibid., p. 16.

53. Peter Knight, The Kennedy Assassination, Cambridge, Cambridge University Press, 2013, p. 4. 
54. Peter Knight, Conspiracy Nation: The Politics of Paranoia in Postwar America, New York, New York University Press, 2002, p. 21.

55. Jodi Dean, Publicity's Secret: How Technoculture Capitalizes on Democracy, Ithaca, NY, Cornell University Press, 2002, p. 52-53.

56. Emma A. Jane et Chris Fleming, Modern Conspiracy: The Importance of Being Paranoid, London, Bloomsbury Publishing, 2014, p. 59.

57. Patrick O’Donnell, Latent Destinies, op. cit., p. 9.

58. "'These violent delights have violent ends' is from Romeo and Juliet act 2 scene 6 when Friar Laurence says the line to Romeo to warn him that a reckless dangerous romance will end the same and this ends up being a theme for Westworld as a whole » Voss, op. cit.

59. «Westworld: A Delos Destination », consulté le 4 février 2018, https://discoverwestworld.com/ $\#$.

60. "Like the real world, I think the agenda is to make a lot of money. " "Westworld Management: What's The Real Agenda? ", Previously.TV Forums, consulté le 11 février 2018, http://forums.previously.tv/topic/48914-westworld-management-whats-the-real-agenda/.

61. Charles Martin, "Friends et la folle théorie de la conspiration Starbucks ! ", Première, 5 juillet 2017, consulté le 11 février 2018, http://www.premiere.fr/Series/News-Series/Friends-et-lafolle-theorie-de-la-conspiration-Starbucks.

62. Lauren Sarner, "Mind-Blowing Fan Theory Links Game of Thrones and Westworld ", Inverse, consulté le 11 février 2018, https://www.inverse.com/article/31740-game-of-thrones-westworldwesteros-crazy-theory-jon-snow.

63. Christopher Hooton, « Westworld season 2 will 'fuck with the metaphysical' ", The Independent, 27 mars 2017, consulté le 11 février 2018, http://www.independent.co.uk/arts-entertainment/tv/ news/westworld-season-2-plot-reddit-storyline-jonathan-nolan-details-news-a7651506.html.

64. La manière dont Donahue a défendu cette théorie est racontée dans Bonar Menninger, Mortal Error: The Shot that Killed JFK, Kansas City, Hunter's Moon Press, 2013.

65. Ibid.

66. Ben Arnold, "The 'truth' about Westworld: seven fan theories to blow your mind ", The Guardian, 30 octobre 2016, consulté le 11 février 2018, http://www.theguardian.com/tv-andradio/2016/oct/30/westworld-wild-fan-theories-about-sci-fi-tv-series.

67. Jan Jagodzinski, Television and Youth Culture, op. cit., p. 25.

68. Voir Jean Baudrillard, La guerre du Golfe n'a pas eu lieu, Paris, Galilée, 1991.

69. Mark Fenster, Conspiracy Theories, op. cit., p. 7.

70. Jan Jagodzinski, Visual Art and Education in an Era of Designer Capitalism: Deconstructing the Oral E ye, New York, Springer, 2010, p. 104.

71. Sur ce sujet, voir par exemple Victor I. Stoichita, L'Effet Pygmalion : Pour une anthropologie historique des simulacres, Paris, Librairie Droz, 2008.

72. Sébastien Lefait, « 'It's not a technological problem we have, it's a human one' - Black Mirror, ou la dystopie intégrée ". L'imaginaire en séries 1, revue Otrante $n^{\circ} 42$ (2017), dir. Hélène Machinal et Elaine Després, Paris, Editions Kimé, 2017, p. 127-44. 


\section{RÉSUMÉS}

Cet article part d'un constat pour en explorer les conséquences. Le constat est simple : Westworld utilise les techniques bien connues du récit complotiste pour créer un univers transmédia mystérieux, dont le public est en permanence incité à percer les secrets. Les conséquences sont bien plus difficiles à sonder : en encourageant une telle lecture complotiste, Westworld étend-elle l'impératif de décryptage paranoïaque au réel dans son ensemble, qui inclut, entre autres, certaines parties de son continuum transmédia? Pour étudier cet aspect de la réception de la série, mon propos s'appuie sur l'analyse d'une vidéo amateur diffusée sur Youtube, où un aficionado de Westworld se livre à une étude conspirationniste de la première saison. Cette analyse permet d'évaluer les conséquences potentielles sur nos sociétés d'une collusion, dont les fans supposent l'existence, entre l'industrie médiatique et la culture du complot.

This paper explores the hidden consequences of a simple observation: Westworld uses the timeproven recipes of conspiracy narrative to create a mystery transmedia universe, the secrets of which viewers are incited to elucidate. The consequences of such a strategy are harder to gauge to what extent does Westworld encourage viewers to read the whole world through the lens of conspiracy, all the more so since parts of the show's transmedia continuum are to be found in the real world? To study this aspect of the show's reception, I analyse a fan video that features a conspiracist reading of the first season. This case study provides an assessment of the potential consequences on current societies of the collusion, as identified by fans of TV shows, between the media industry and the current popularity of conspiracy thinking.

\section{INDEX}

Keywords : Conspiracy, paranoia, secrecy, decoding, media reception, influence of cultural productions

Mots-clés : Complot, paranoïa, secret, décodage, réception médiatique, influence des productions culturelles

\section{AUTEUR}

\section{SÉBASTIEN LEFAIT}

Sébastien Lefait est professeur d'études américaines et médiatiques à l'université Paris 8 . Ses travaux de recherche portent sur l'adaptation des pièces de Shakespeare, sur les sociétés de surveillance et leur représentation à l'écran, ainsi que sur la dimension réflexive des séries télévisées. Il a notamment publié In Praise of Cinematic Bastardy (2012; co-dirigé avec Philippe Ortoli), Surveillance on Screen: Monitoring Contemporary Films and Television Programs (2013), et La question raciale dans les séries américaines (2014; coécrit avec Olivier Esteves). Sa recherche actuelle porte sur les relations entre terrorisme et fiction, sur la place de la téléréalité dans la culture américaine, et sur le traitement de la question raciale dans quelques séries télévisées récentes. 
Sébastien Lefait is Full Professor of American Studies and Media Studies at Université Paris 8. His research focuses on the adaptation of Shakespeare's plays, on surveillance and its representation on screen, and on the reflexive dimension of TV series. His published works include In Praise of Cinematic Bastardy (coedited with Philippe Ortoli, 2012); Surveillance on Screen: Monitoring Contemporary Films and Television Programs (2013), and La question raciale dans les séries américaines (co-written with Olivier Esteves, 2014). His current research focuses on the relationship between terrorism and fiction; on reality TV within American culture, and on the way recent TV series have dealt with racial issues. 